\title{
Rankl Inhibitor Enhances Bone Modeling after Surgical Fixation for Atypical Femoral Fracture: A Case Report and Review of Management Options
}

\author{
Ong Art Phruetthiphat*, Suphot Phruetthiphat, Sasawat Tonvichien and Thawee Songpatanasilp \\ Phramongkutklao Hospital and College of Medicine, Thailand \\ *Corresponding author: Ong Art Phruetthiphat, Thailand
}

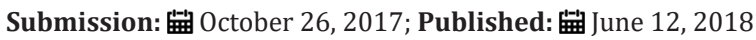

\begin{abstract}
Introduction: Atypical femoral fracture (AFF) may be associated with prolonged bisphosphonate. Any bisphosphonate after surgical fixation for AFF should not prescribe in this group of patients. Additionally, animal studies have shown that bisphosphonates suppress bone formation by lining cells, that is, bone modeling [1,2]. RANKL-inhibitor is one of the most effective treatment for osteoporosis after surgical intervention for AFF without bone modeling suppression.

Case presentation: A 78 years old female with prolonged oral bisphosphonate longer than 5 years sustained an atypical femoral fracture on left thigh by simple fall. She underwent surgical fixation with broad dynamic compression plate. One week after surgery, she fell again and she came with re-fracture around plate fixation. Fixation failure was managed by distal femoral Locking Compression Plate (LCP) with cable fixation and followed by RANKL inhibitor 2 weeks after surgery. Fracture completely united by 1 year after 2 doses of denosuma band patient can walk with a small limitation by cane.
\end{abstract}

Conclusion: RANKL inhibitor may be useful for osteoporotic treatment with enhancing bone modeling after surgical intervention for atypical femoral fracture.

Keywords: RANKL inhibitor; Atypical femoral fracture; Surgical fixation; Bone modeling

\section{What We Learn from this Article?}

Atypical femoral fracture is associated with prolonged bisphosphonate. Moreover, anti-resorptive drug for osteoporotic treatment may interfere bone modeling and may suppress bone turnover. However, RANKL-inhibitor may enhance bone modeling after surgical fixation for atypical femoral fracture.

\section{Introduction}

Denosumab is an antibody against the RANK-ligand (RANKL) and by neutralizing RANKL osteoclast recruitment, activity and life span is reduced. Treatment with denosumab has led to very impressive increases in bone mass, especially at sites with a high content of cortical bone [3]. Furthermore, the increases in bone mass seem to continue because bone turnover is continuously suppressed while modeling-based bone formation continues unabated [4,5]. We report a unique case of increasing bone modeling treated by denosumab after surgical fixation for atypical femoral fracture [6].

\section{Case Report}

A 78 years old female with prolonged oral bisphosphonate for 7 years sustained an atypical femoral fracture on left thigh by simple fall (Figure 1). She underwent surgical fixation with broad dynamic compression plate from private hospital (Figure 2). One week after surgery, she fell again and she came with re-fracture around plate fixation (Figure 3). Fixation failure was managed by distal femoral Locking Compression Plate (LCP) (Figure 4) and followed by RANKL inhibitor 2 weeks after surgery. There was a progression of mineralization at 3,6, and 9 months follow up (Figure 5 \& 6). Fracture completely united by 1 year (Figure 7 \& 8) and patient can walk with a small limitation by cane.

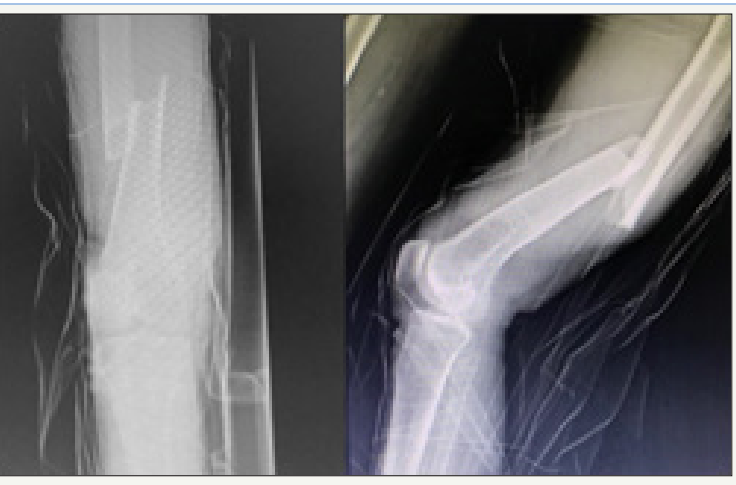

Figure 1 


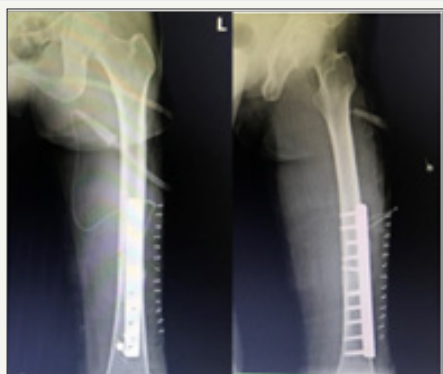

Figure 2

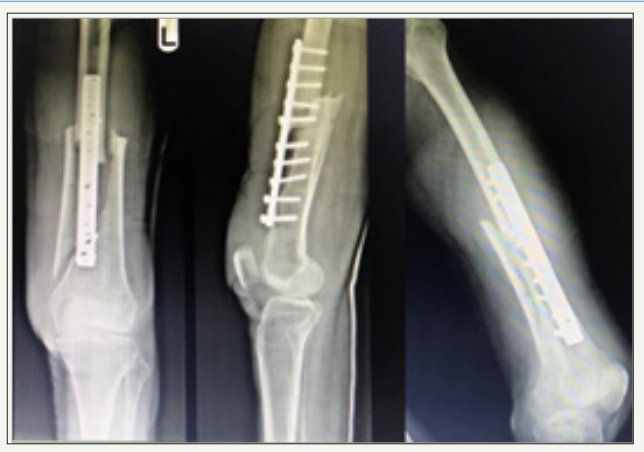

Figure 3

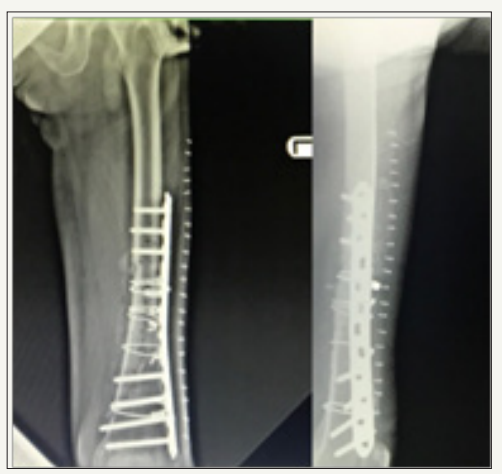

Figure 4

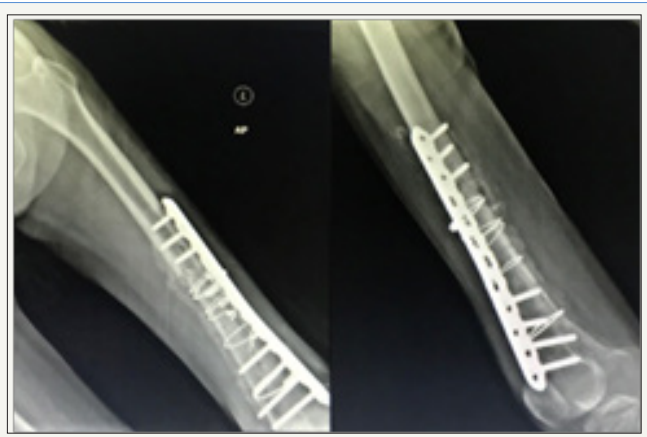

Figure 5

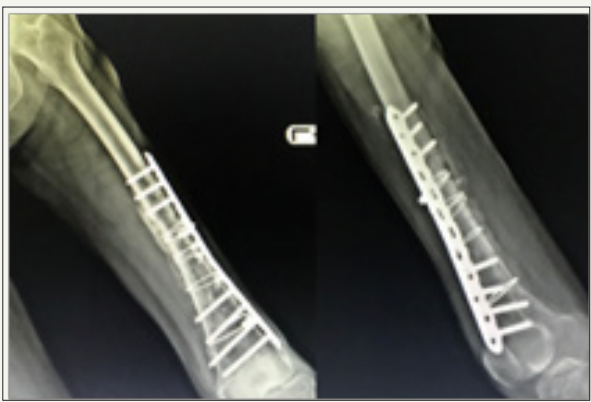

Figure 6

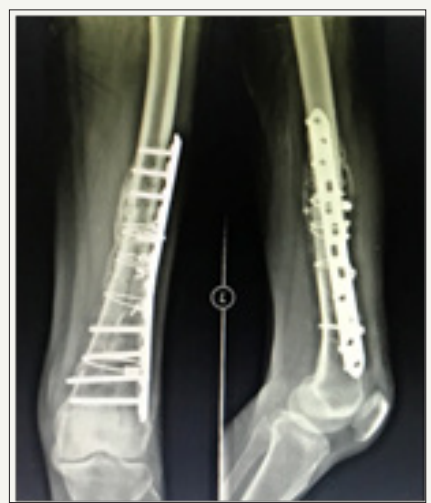

Figure 7

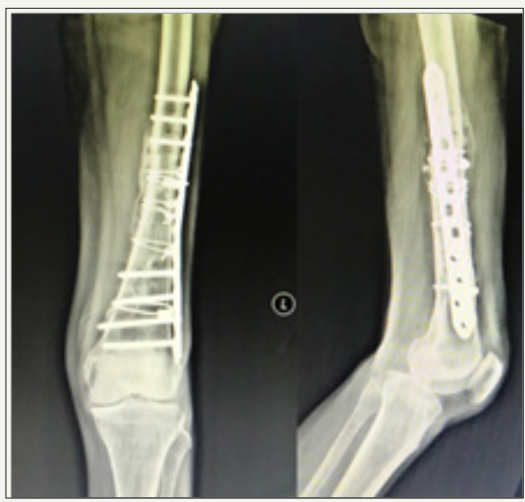

Figure 8

\section{Discussion}

Patient had a history of prolonged oral bisphosphonate (BPs; Actonel) for 7 years and she sustained atypical femoral fracture on left thigh from low energy trauma. This case had two common pitfalls in orthopedics:

1. Ignorance of bone quality along femoral shaft (Atypical femoral fracture) so orthopedist just applied Dynamic Compression Plate (DCP) representing inadequate fixation for osteoporotic bone;

2. Error in fall prevention, patient fell down again and she sustained re-fracture along previous plate. This consequence complication can avoid by recognition of AFF in all patients with prolonged history of bisphosphonate and closely take care for fall prevention by FLS management.

Moreover, osteoporosis therapy after revision by LCP and cable fixation is also important for increasing bone mass density and for further reduction of fracture risk. We prescribed RANKL inhibitor for this patient because she had an evidence of AFF associated with oral BPs. Additionally, an evidence by B. Langdahl and S.Ferrari suggested us for denosumab post operatively because it inhibits bone remodeling but is permissive for modeling at cortex. The reason why we did not apply teriparatidefor AFF because of an economic issue in our country.

\section{Conclusion}

RANKL inhibitor is an alternative option for osteoporosis therapy with promoting bone modeling after surgical intervention for atypical femoral fracture. 


\section{References}

1. Gasser J, Kneissel M, Thomsen J, Mosekilde L (2000) PTH and interactions with bisphosphonates. J Musculoskelet Neuronal Interact 1(1): 53-56.

2. Gasser J, Green J (2006) Chronic subcutaneous, but not single intravenous, dosing of rats with bisphosphonates results in reduced anabolic response to PTH. J Bone Miner Res Suppl 1: F386.

3. Cummings S, Martin SJ, McClung M, Siris E, Eastell R, et al. (2009) Denosumab for prevention of fractures in postmenopausal women with osteoporosis. N Engl J Med 361(8): 756-765.
4. Bone HG, Chapurlat R, Brandi M, Brown J, Czerwinski E, et al. (2013) The effect of three or six years of denosumab exposure in women with postmenopausal osteoporosis: results from the FREEDOM extension. J Clin Endocrinol Metab 98(11): 4483-4492.

5. Ominsky MS, Libanati C, Niu QT, Boyce RW, Kostenuik PJ, et al. (2015) Sustained modeling-based bone formation during adulthood in cynomolgus monkeys may contribute to continuous BMD gains with denosumab. J Bone Miner Res 30(7): 1280-1289.

6. Langdahl B, Ferrari S, Dempster DW (2016) Bone modeling and remodeling: potential as therapeutic targets for the treatment of osteoporosis. Ther Adv Musculoskel Dis 8(6): 225-235.
Creative Commons Attribution 4.0 International License

For possible submissions Click Here

\section{Submit Article}

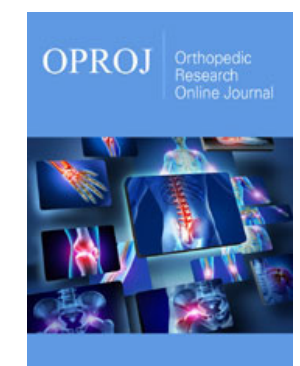

\section{Orthopedic Research Online Journal}

\section{Benefits of Publishing with us}

- High-level peer review and editorial services

- Freely accessible online immediately upon publication

- Authors retain the copyright to their work

- Licensing it under a Creative Commons license

- Visibility through different online platforms 